SFB/CPP-12-93

TTP12-45

LPN12-127

\title{
Higgs boson production at the LHC: NNLO partonic cross sections through order $\epsilon$ and convolutions with splitting functions to $\mathrm{N}^{3} \mathrm{LO}$
}

\author{
Maik Höschele, Jens Hoff, Alexey Pak, \\ Matthias Steinhauser, Takahiro Ueda \\ Institut für Theoretische Teilchenphysik \\ Karlsruhe Institute of Technology (KIT) \\ 76128 Karlsruhe, Germany
}

\begin{abstract}
We consider Higgs boson production at hadron colliders in the gluon fusion channel and compute higher order terms in the regularization parameter $\epsilon$. In particular, the next-to-next-to-leading order cross section is evaluated including order $\epsilon$ terms. These results are used to compute all convolutions with the splitting functions entering the next-to-next-to-next-to-leading order cross section.
\end{abstract}

PACS numbers: 12.38.Bx, 14.80.Bn 


\section{Introduction}

After the recent discovery of a new boson at the Large Hadron Collider (LHC) at CERN [1, 2] one has to clarify the question whether it is the Standard Model (SM) Higgs boson or a new particle of an enlarged theory. For this purpose it is important to study in detail its production and decay processes. In the SM, but also in many extensions, the largest production cross section for a Higgs boson is given by the gluon fusion sub-process which, however, also has a large uncertainty. The uncertainties arise mainly from the parton distribution functions (PDFs) and from unknown higher order corrections. The latter amount to about $10 \%$ although perturbative next-to-next-to-leading order (NNLO) QCD and NLO electroweak corrections have been computed in the recent years and are thus available for theory predictions (see Refs. $[3,4]$ for comprehensive reviews). In view of the expected experimental precision reached by the LHC experiments it is desirable to take the next step and compute the $\mathrm{N}^{3} \mathrm{LO}$ QCD corrections to the gluon fusion process. In this letter we contribute important building blocks to this enterprise by computing the lower order partonic cross sections to higher order in $\epsilon=(4-D) / 2$, where $D$ is the space-time dimension, and by providing results for their convolution with the splitting functions. In our calculation we will make use of the results for the master integrals entering the NNLO calculation. In Refs. [5,6] results for the $\epsilon$ expansion are provided which are sufficient for the $\mathrm{N}^{3} \mathrm{LO}$ predictions.

The starting point for the evaluation of the cross section is the effective five-flavour Lagrange density

$$
\mathcal{L}_{Y, \text { eff }}=-\frac{H^{0}}{v^{0}} C_{1}^{0} \mathcal{O}_{1}^{0}+\mathcal{L}_{Q C D}^{(5)}
$$

where $\mathcal{L}_{Q C D}^{(5)}$ is the usual QCD part with five massless quarks, $H^{0}$ denotes the Higgs boson, $v^{0}$ its vacuum expectation value and $C_{1}^{0}$ is the matching coefficient between the full and the effective theory. $G_{\mu \nu}^{0}$ is the gluonic field strength tensor constructed from fields and couplings already present in $\mathcal{L}_{Q C D}^{(5)}$. The superscript " 0 " denotes the bare quantities. Note that the renormalization of $H^{0} / v^{0}$ is of higher order in the electromagnetic coupling constant. In Eq. (1) the operator $\mathcal{O}_{1}^{0}$ is given by

$$
\mathcal{O}_{1}^{0}=\frac{1}{4} G_{\mu \nu}^{0} G^{0, \mu \nu}
$$

As shown in Refs. [5,7-11], through NNLO the results obtained in the effective-theory approach agree to good accuracy with the ones of the full-theory calculation. We assume that this feature also holds at $\mathrm{N}^{3} \mathrm{LO}$.

The outline of the paper is as follows: in the next two Sections we discuss in detail the splitting functions and the partonic cross sections up to NNLO. The procedure to compute the convolution integrals is described in Section 4 where also some sample results are given. Analytical results for all convolutions are provided in a Mathematica file which can be downloaded from [12]. In Section 5 we summarize our findings. 


\section{Splitting functions}

In general, the sum of the renormalized real and the virtual contributions of a given partonic sub-process is not finite in the limit $\epsilon \rightarrow 0$. The remaining poles originate from the collinear divergences in the initial state. For example, the initial gluon may split into a quark-antiquark pair and the quark participates in the Higgs boson production. It also may happen that a quark in the initial state radiates a gluon before participating in the hard scattering process.

The correct treatment of the singularities is achieved by the convolution of the partonic cross section with the splitting functions $P_{i j}(x)$ describing the probability of parton $j$ to emit a parton $i$ with the fraction $x$ of its initial energy. The perturbative expansion is given by

$$
P_{i j}(x)=\delta_{i j} \delta(1-x)+\frac{\alpha_{s}^{(5)}}{\pi} P_{i j}^{(1)}(x)+\left(\frac{\alpha_{s}^{(5)}}{\pi}\right)^{2} P_{i j}^{(2)}(x)+\left(\frac{\alpha_{s}^{(5)}}{\pi}\right)^{3} P_{i j}^{(3)}(x)+\ldots
$$

where the analytical results for $P_{i j}^{(k)}(x)$ can be found in Refs. [13-16]. It is common practice to refer to $P_{i j}^{(k)}(x)$ as the $k$-loop splitting function.

We have written the expansion in Eq. (3) in terms of $\alpha_{s}^{(5)}$, the renormalized coupling in the effective theory with decoupled top quark and $n_{l}=5$ massless quarks.

In our calculation we use the splitting functions defined in the $\overline{\mathrm{MS}}$ scheme. This is consistent with the definition of the parton distribution functions (PDFs) which absorb the non-perturbative and non-singular features of the initial state.

For definiteness let us present the explicit results for the one-loop splitting functions which $\operatorname{read}^{1}$

$$
\begin{aligned}
& P_{q q}^{(1)}=-\frac{2}{3}-\frac{2 x}{3}+\delta(1-x)+\frac{4}{3}\left[\frac{1}{1-x}\right]_{+}, \\
& P_{q g}^{(1)}=\frac{1}{4}-\frac{x}{2}+\frac{x^{2}}{2}, \\
& P_{g q}^{(1)}=-\frac{4}{3}+\frac{4}{3 x}+\frac{2 x}{3}, \\
& P_{g g}^{(1)}=-6+\frac{3}{x}+3 x-3 x^{2}+\left(\frac{11}{4}-\frac{n_{l}}{6}\right) \delta(1-x)+3\left[\frac{1}{1-x}\right]_{+},
\end{aligned}
$$

where $n_{l}$ is the number of active flavours. As far as the two-loop splitting function $P_{q q}^{(2)}$ is concerned we use $P_{q q}=P_{\mathrm{ns}}^{+}+P_{\mathrm{ps}}[16]$ where the two-loop terms of $P_{\mathrm{ns}}^{+}$and $P_{\mathrm{ps}}$ are given in Eq. (4.6) of Ref. [15] and Eq. (4.7) of Ref. [16], respectively. The analytical result reads

$$
P_{q q}^{(2)}=\frac{1}{2}-\frac{143 x}{18}+\pi^{2}\left(\frac{4}{27}+\frac{5 x}{27}+\frac{1}{27(1+x)}\right)+n_{l}\left(-\frac{19}{27}+\frac{20}{27 x}+\frac{65 x}{27}-\frac{56 x^{2}}{27}\right)
$$

\footnotetext{
${ }^{1}$ Note that our definition of $P_{q g}^{(k)}$ differs by a factor $2 n_{l}$ from that of Ref. [16].
} 


$$
\begin{aligned}
& +\left[-\frac{17}{18}+\frac{7}{3(1-x)}-\frac{11 x}{6}+n_{l}\left(\frac{4}{9}-\frac{2}{9(1-x)}+\frac{16 x}{9}+\frac{8 x^{2}}{9}\right)\right] \mathrm{H}_{0}(x) \\
& +\left(-\frac{8}{9}+\frac{16}{9(1-x)}-\frac{8 x}{9}\right) \mathrm{H}_{2}(x)+\left(-\frac{2}{9}+\frac{2 x}{9}+\frac{4}{9(1+x)}\right) \mathrm{H}_{-1,0}(x) \\
& +\left[-\frac{4}{3}+\frac{2}{(1-x)}-\frac{14 x}{9}-\frac{2}{9(1+x)}+n_{l}\left(-\frac{2}{3}-\frac{2 x}{3}\right)\right] \mathrm{H}_{0,0}(x) \\
& +\left(-\frac{8}{9}+\frac{16}{9(1-x)}-\frac{8 x}{9}\right) \mathrm{H}_{1,0}(x)+\left(\frac{67}{9}-\frac{\pi^{2}}{3}-n_{l} \frac{10}{27}\right)\left[\frac{1}{1-x}\right]_{+} \\
& +\left[\frac{7}{8}+\frac{7 \pi^{2}}{18}-\frac{\zeta(3)}{3}+n_{l}\left(-\frac{1}{36}-\frac{\pi^{2}}{27}\right)\right] \delta(1-x),
\end{aligned}
$$

where $\zeta(n)$ is Riemann's zeta function. The remaining two-loop splitting functions can be found in Eqs. (4.8), (4.9) and (4.10) and the three-loop ones in Eqs. (4.14) and (4.15) of Ref. [16]. Note that at the three-loop level only $P_{g q}^{(3)}$ and $P_{g g}^{(3)}$ are needed.

\section{Partonic cross sections}

The renormalized partonic cross section at $\mathrm{N}^{3} \mathrm{LO}$ receives contributions from convolutions of splitting functions with the renormalized lower order partonic cross sections. For this purpose the latter needs to be expanded to higher order in $\epsilon$ since the convolutions with $P_{i j}^{(k)}(x)$ come along with poles in $\epsilon .^{2}$ Furthermore, $\alpha_{s}$ and the operator $\mathcal{O}_{1}$ have to be renormalized which means that the LO, NLO and NNLO partonic cross sections are multiplied by contributions containing $1 / \epsilon$ poles and thus the $\epsilon$ expansion needs to be sufficiently deep.

We have performed two independent calculations of the partonic cross sections. On the one hand we extended the full-theory calculation of Ref. [10] to one order higher in $\epsilon$ and have computed the leading term in the heavy-top quark mass expansion. On the other hand we have implemented the effective-theory Feynman rules (cf. Eq. (1)) and evaluated the partonic cross sections of all sub-processes. Of course, the NLO and the NNLO results present in the literature [17-22] could be reproduced. The $\mathcal{O}\left(\epsilon^{2}\right)$ terms at NLO and the $\mathcal{O}(\epsilon)$ terms at NNLO are new.

From Eq. (1) it is clear that the partonic cross sections contain the two factors, the matching coefficient $C_{1}$ and the cross section computed using the effective Higgs-gluon coupling contained in $H^{0} \mathcal{O}_{1}^{0}$. Thus, for the renormalized cross section we can write

$$
\hat{\sigma}_{i j}=C_{1}^{2} \tilde{\sigma}_{i j}
$$

\footnotetext{
${ }^{2}$ We refer to $[17,18]$ for a nice description of the procedure in case of NNLO Higgs boson production. In particular, in Ref. [17] one finds explicit formulae showing how the convolutions of splitting functions and partonic cross sections enter the renormalized result.
} 
with $C_{1}=-\alpha_{s}^{(5)} /(3 \pi)\left(1+\mathcal{O}\left(\alpha_{s}\right)\right)$. In the remainder of the paper we will only consider $\tilde{\sigma}_{i j}$, which we refer to as the "reduced partonic cross section", since only this quantity enters the convolutions with the splitting functions; the multiplication with the finite factor $C_{1}^{2}$ can be done at the very end. We note that the matching coefficient $C_{1}$ has been computed to three- $[23,24]$ and four-loop order $[23,25,26]$, respectively.

In analogy to Eq. (3) we write the perturbative expansion of the reduced partonic cross section as

$$
\tilde{\sigma}_{i j}(x)=A\left[\tilde{\sigma}_{i j}^{(0)}(x)+\frac{\alpha_{s}^{(5)}}{\pi} \tilde{\sigma}_{i j}^{(1)}(x)+\left(\frac{\alpha_{s}^{(5)}}{\pi}\right)^{2} \tilde{\sigma}_{i j}^{(2)}(x)+\ldots\right],
$$

with $A=G_{F} \pi /(32 \sqrt{2})$ and $x=M_{h}^{2} / s$. The dependence on $\mu^{2} / M_{h}^{2}$, where $\mu$ represents the renormalization and the factorization scale, is suppressed. At NLO we have $i, j \in\{g, q, \bar{q}\}$ and at the NNLO the possible initial states are given by $g g, q g, \bar{q} g, q \bar{q}, q q$, or $q q^{\prime}$, where $q$ and $q^{\prime}$ stand for (different) massless quark flavours. ${ }^{3}$ The computation of the $\mathrm{N}^{3} \mathrm{LO}$ contributions requires that $\tilde{\sigma}^{(k)}$ is known to order $\epsilon^{3-k}$.

In order to fix the notation we provide the explicit results for $\tilde{\sigma}_{i j}^{(0)}$ and $\tilde{\sigma}_{q \bar{q}}^{(1)}$ which are given by

$$
\begin{aligned}
\tilde{\sigma}_{i j}^{(0)}= & \delta_{i g} \delta_{j g} \frac{\delta(1-x)}{1-\epsilon} \\
\tilde{\sigma}_{q \bar{q}}^{(1)}= & \frac{32}{27}(1-x)^{3}\left\{1+\epsilon\left(\frac{2}{3}+\mathrm{H}_{0}\left(x_{\mu H}\right)-2 \mathrm{H}_{0}(1-x)+\mathrm{H}_{0}(x)\right)\right. \\
& +\epsilon^{2}\left[\frac{13}{9}-\frac{\pi^{2}}{4}+\frac{1}{2}\left(\mathrm{H}_{0}\left(x_{\mu H}\right)\right)^{2}+2\left(\mathrm{H}_{0}(1-x)\right)^{2}+\mathrm{H}_{0}(1-x)\left(-\frac{4}{3}-2 \mathrm{H}_{0}(x)\right)\right. \\
& \left.\left.+\frac{2}{3} \mathrm{H}_{0}(x)+\frac{1}{2}\left(\mathrm{H}_{0}(x)\right)^{2}+\mathrm{H}_{0}\left(x_{\mu H}\right)\left(\frac{2}{3}-2 \mathrm{H}_{0}(1-x)+\mathrm{H}_{0}(x)\right)\right]\right\}
\end{aligned}
$$

with $x_{\mu H}=\mu^{2} / M_{H}^{2}$. The other results for the partonic cross sections are significantly larger and are given in computer readable form in Ref. [12].

\section{Convolutions of partonic cross sections and split- ting functions}

The convolution of functions $f$ and $g$ that enter $P_{i j}^{(k)}(x)$ and $\hat{\sigma}_{i j}$ is defined as

$$
[f \otimes g](x)=\int_{0}^{1} \mathrm{~d} x_{1} \mathrm{~d} x_{2} \delta\left(x-x_{1} x_{2}\right) f\left(x_{1}\right) g\left(x_{2}\right) .
$$

\footnotetext{
${ }^{3}$ It is understood that ghosts are always considered together with gluons.
} 
Both the results for the partonic cross sections (see previous Section) and the splitting functions include combinations of HPLs up to weight four with factors $1 / x, 1 /(1-x)$, and $1 /(1+x)$, and the generalized functions $\delta(1-x)$ and $\left[\frac{\ln ^{k}(1-x)}{1-x}\right]_{+}$. In the following we briefly describe our approach to compute the convolutions of such functions. A detailed description can also be found in Appendix B of Ref. [5].

1. First, we transform the convoluted expressions to Mellin space via

$$
M_{n}[f(x)]=\int_{0}^{1} \mathrm{~d} x x^{n-1} f(x) .
$$

This transformation turns convolutions into products due to the identity

$$
M_{n}[[f \otimes g](x)]=M_{n}[f(x)] M_{n}[g(x)] .
$$

A comprehensive discussion of the Mellin transform and the list of all Mellin images appearing in the calculation of the NNLO Higgs boson production rate can be found in Refs. [27,28]. Mellin transforms of HPLs and their derivatives can be conveniently expressed in terms of harmonic sums $[29,30]$. In our calculation, we used the FORM package harmpol [31] to perform the transformation.

2. Second, we prepare a table of Mellin transforms of HPLs to certain maximum weight. For example, the table through weight one reads

$$
\begin{aligned}
M_{n}[1] & =\frac{1}{n} \\
M_{n}\left[\mathrm{H}_{0}(x)\right] & =-\frac{1}{n^{2}}, \\
M_{n}\left[\mathrm{H}_{1}(x)\right] & =\frac{\mathrm{S}_{1}(n)}{n}, \\
M_{n}\left[\mathrm{H}_{-1}(x)\right] & =-\frac{(-1)^{n}}{n}\left(\mathrm{~S}_{-1}(n)+\ln 2\right)+\frac{\ln 2}{n},
\end{aligned}
$$

where $\mathrm{H}_{i}(x)$ are the HPLs of weight one and $\mathrm{S}_{i}(n)$ are the harmonic sums of weight one. Mellin transforms of HPLs of higher weights produce harmonic sums of higher weights and transcendental numbers originating from HPLs evaluated at $x=1$.

3. Third, we prepare a table of Mellin transforms of "regularized derivatives" of HPLs in the table above as follows:

$$
\begin{aligned}
& M_{n}\left[\hat{\partial}_{x} 1\right]=1, \\
& M_{n}\left[\hat{\partial}_{x} f(x)\right]=R[f(x)]-(n-1) M_{n-1}[f(x)],
\end{aligned}
$$

where

$$
R\left[g_{k}(x) \ln ^{k}(1-x)+g_{k-1}(x) \ln ^{k-1}(1-x)+\ldots+g_{0}(x)\right]=g_{0}(1)
$$


with $g_{j}(1) \neq 0 \quad \forall j>0$.

This definition allows us to establish relations between the regularized derivatives of HPLs and the "common" generalized functions. In particular,

$$
\begin{aligned}
\hat{\partial}_{x} 1 & =\delta(1-x) \\
\hat{\partial}_{x} \mathrm{H}_{1}(x) & =\left[\frac{1}{1-x}\right]_{+}, \\
\hat{\partial}_{x} \mathrm{H}_{11}(x) & =-\left[\frac{\ln (1-x)}{1-x}\right]_{+}, \\
\hat{\partial}_{x} \mathrm{H}_{111}(x) & =\frac{1}{2}\left[\frac{\ln ^{2}(1-x)}{1-x}\right]_{+}, \\
\hat{\partial}_{x} \mathrm{H}_{101}(x) & =\frac{\pi^{2}}{6}\left[\frac{1}{1-x}\right]_{+}+\frac{\mathrm{H}_{01}(x)-\zeta_{2}}{1-x} .
\end{aligned}
$$

Note that if $f(x)$ is not divergent at $x=1$, its regularized derivative Eq. (14) reduces to the usual derivative:

$$
M_{n}\left[\frac{d}{d x} f(x)\right]=\left.x^{n-1} f(x)\right|_{0} ^{1}-(n-1) M_{n-1}[f(x)] .
$$

For example,

$$
\begin{aligned}
\hat{\partial}_{x} \mathrm{H}_{-1}(x) & =\frac{d}{d x} \mathrm{H}_{-1}(x)=\frac{1}{1+x}, \\
\hat{\partial}_{x} \mathrm{H}_{-1,1}(x) & =\frac{d}{d x} \mathrm{H}_{-1,1}(x)=\frac{\mathrm{H}_{1}(x)}{1+x} .
\end{aligned}
$$

Thus, it is not necessary to separately consider Mellin transforms of all functions appearing in the derivatives of HPLs, such as $\frac{H_{\ldots} \ldots(x)}{1+x},\left[\frac{H_{\ldots} \ldots(x)}{1-x}\right]_{+}$and so on. Rather it is sufficient to consider Mellin transforms of regularized derivatives in case Mellin transforms for generalized functions are needed. For illustration we list the Mellin transforms of regularized derivatives of HPLs to weight one

$$
\begin{aligned}
M_{n}\left[\hat{\partial}_{x} 1\right] & =1 \\
M_{n}\left[\hat{\partial}_{x} \mathrm{H}_{0}(x)\right] & =\frac{1}{n-1}, \\
M_{n}\left[\hat{\partial}_{x} \mathrm{H}_{1}(x)\right] & =-\mathrm{S}_{1}(n-1), \\
M_{n}\left[\hat{\partial}_{x} \mathrm{H}_{-1}(x)\right] & =(-1)^{n-1} \mathrm{~S}_{-1}(n-1)+(-1)^{n-1} \ln 2 .
\end{aligned}
$$

4. Finally, we prepare and solve the system of linear equations and thus perform the inverse Mellin transform. Combining the tables for Mellin transforms of HPLs as in Eqs. (12) and the regularized derivatives of HPLs as in Eqs. (19) one obtains a 
system of linear equations for terms of the form $1 / n^{k}, \mathrm{~S}_{\ldots}(n) / n^{k}$ and $(-1)^{n} \mathrm{~S} \ldots(n) / n^{k}$ which can be used in order to find the inverse Mellin transforms. In principle, the expression in the Mellin space may have other terms, whose inverse transform cannot be determined from the system, but all such terms cancel in the real calculation.

The above steps have been implemented in a Mathematica program which uses the HPL package $[32,33]$ and can compute all the necessary convolutions.

As an important cross check, we have performed all the convolution integrals also numerically for some specific value of $x$. In case no plus distribution is present in the integrand it is straightforward to perform the numerical integration. For convolution integrals involving one or more plus distributions, the cancellation of the singular behaviour among terms can be quite involved. The problem can be solved by introducing auxiliary regularizations [34]

$$
\frac{\ln ^{n}(1-x)}{1-x} \rightarrow \lim _{\eta \rightarrow 0} \lim _{a \rightarrow 1} \frac{1}{\eta^{n}} \frac{\partial^{n}}{\partial a^{n}}(1-x)^{-1+a \eta},
$$

for evaluating integrals of the individual terms. After performing the integrals by using $\delta$ functions in the definition of the convolutions one rescales the integration variables such that the integration domain becomes the unit hypercube. The singularities come from both the lower and the upper limit of the integration as well as from the overall $(1-x)^{-1+a \eta}$ factors which give $\delta$ functions and plus distributions in the result of the convolution, after applying

$$
\lambda^{-1+a \eta}=\frac{\delta(\lambda)}{a \eta}+\sum_{j=0}^{\infty} \frac{(a \eta)^{j}}{j !}\left[\frac{\ln ^{j}(\lambda)}{\lambda}\right]_{+} .
$$

In the next step one differentiates the result with respect to $a$, takes the limit $a \rightarrow 1$, and finally expands in $\eta$. All pole terms $1 / \eta^{k}$ should cancel among the integrals and hence the leading term gives the result of the convolution. The remaining integrals have no divergences and can be performed numerically.

As a further welcome check we have compared our results with the explicit expressions for the convolutions given in the Appendix of Ref. [35]. In particular we found complete agreement with Eqs. (C.28) to (C.31) which involve convolutions of two plus distributions.

In Tab. 1 we classify the convolutions which are required for the $\mathrm{N}^{3} \mathrm{LO}$ calculation according to the number of nested convolutions. Contributions where only one convolution is involved already enter the NLO and the NNLO results. At the $\mathrm{N}^{3} \mathrm{LO}$, the LO, NLO and NNLO partonic cross section has to be convoluted with three-, two- and one-loop splitting functions, respectively. Two convolution integrals are only present at NNLO and $\mathrm{N}^{3} \mathrm{LO}$ and three convolutions only at $\mathrm{N}^{3} \mathrm{LO}$. In the latter case, however, only one-loop splitting functions and the leading order cross section enter. Since the latter is proportional to $\delta(1-x)$, only two non-trivial integrals remain.

The right-most column in Tab. 1 defines the name of the file where the analytical results can be found. At NLO there are only 2 non-zero convolutions, at NNLO there are already 


\begin{tabular}{|c|c|c|}
\hline convolution & order & file name \\
\hline$\left(\tilde{\sigma}_{i j}^{(n)} / x\right) \otimes P_{j k}^{(r)}$ & $\begin{array}{c}\mathrm{N}^{(n+r)} \mathrm{LO} \\
n=0,1,2 \\
r=1,2,3\end{array}$ & Stil $\langle\mathrm{n}\rangle\langle i j\rangle-\mathrm{P}\langle\mathrm{r}\rangle\langle j \mathrm{k}\rangle \cdot \mathrm{m}$ \\
\hline $\begin{array}{l}\left(\tilde{\sigma}_{i j}^{(n)} / x\right) \otimes P_{j k}^{(r)} \otimes P_{k l}^{(s)} \\
P_{i l}^{(s)} \otimes\left(\tilde{\sigma}_{i j}^{(n)} / x\right) \otimes P_{j k}^{(r)}\end{array}$ & $\begin{array}{c}\mathrm{N}^{(n+r+s)} \mathrm{LO} \\
n=0,1 \\
r, s=1,2\end{array}$ & 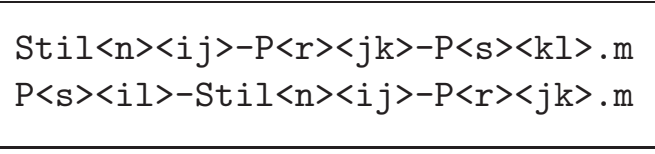 \\
\hline $\begin{array}{l}\left(\tilde{\sigma}_{i j}^{(0)} / x\right) \otimes P_{j k}^{(1)} \otimes P_{k l}^{(1)} \otimes P_{l m}^{(1)} \\
P_{i m}^{(1)} \otimes\left(\tilde{\sigma}_{i j}^{(0)} / x\right) \otimes P_{j k}^{(1)} \otimes P_{k l}^{(1)}\end{array}$ & $\mathrm{N}^{3} \mathrm{LO}$ & $\begin{array}{l}\text { Stil } 0<i j>-P 1<j k>-P 1<k l>-P 1<l m>. m \\
P 1<i m>- \text { Stil } 0<i j>-P 1<j k>-P 1<k l>. m\end{array}$ \\
\hline
\end{tabular}

Table 1: Classifications of the convolutions needed up to order $\mathrm{N}^{3} \mathrm{LO}$ where $i, j, k, l, m \in$ $\left\{g, q, \bar{q}, q^{\prime}\right\}$. As far as splitting functions are concerned there is no difference between " $q$ ", " $\bar{q} "$ and " $q$ ". Furthermore, we have $\tilde{\sigma}_{i j}^{(n)}=\tilde{\sigma}_{j i}^{(n)}$.

18 and at $\mathrm{N}^{3} \mathrm{LO}$ altogether 82 convolutions of partonic cross sections with up to three splitting functions. All the results can be downloaded from [12] where for convenience we also provide intermediate results for the convolutions involving only splitting functions. In Appendix A we provide a list assigning the convolutions to the file names. Note that not all results are shown which are identical due to symmetry.

The graphical representations of the five different types of convolutions given in Tab. 1 are displayed in Fig. 1.

Let us at the end list some convolutions which appear as building blocks in the course of our calculation.

$$
\begin{aligned}
& {\left[\left[\frac{\ln ^{3}(1-x)}{1-x}\right]_{+} \otimes\left[\frac{1}{1-x}\right]_{+}\right]^{2}=\frac{6}{1-x}\left(\mathrm{H}_{1,1,2}(x)+\mathrm{H}_{1,2,1}(x)+\mathrm{H}_{2,1,1}(x)+\mathrm{H}_{1,1,1,0}(x)\right)} \\
& \quad+\frac{5}{4}\left[\frac{\ln ^{4}(1-x)}{1-x}\right]_{+}-\frac{\pi^{2}}{2}\left[\frac{\ln ^{2}(1-x)}{1-x}\right]_{+}+6 \zeta(3)\left[\frac{\ln (1-x)}{1-x}\right]_{+}-\frac{\pi^{4}}{15}\left[\frac{1}{1-x}\right]_{+} \\
& \quad+6 \zeta(5) \delta(1-x), \\
& \left.\left[\frac{\ln ^{4}(1-x)}{1-x}\right]_{+} \otimes\left[\frac{1}{1-x}\right]_{+}\right]=-\frac{24}{1-x}\left(\mathrm{H}_{1,1,1,2}(x)+\mathrm{H}_{1,1,2,1}(x)+\mathrm{H}_{1,2,1,1}(x)\right. \\
& \left.\quad+\mathrm{H}_{2,1,1,1}(x)+\mathrm{H}_{1,1,1,1,0}(x)\right)+\frac{6}{5}\left[\frac{\ln ^{5}(1-x)}{1-x}\right]_{+}-\frac{2 \pi^{2}}{3}\left[\frac{\ln ^{3}(1-x)}{1-x}\right]_{+} \\
& \quad+12 \zeta(3)\left[\frac{\ln ^{2}(1-x)}{1-x}\right]_{+}-\frac{4 \pi^{4}}{15}\left[\frac{\ln (1-x)}{1-x}\right]_{+}+24 \zeta(5)\left[\frac{1}{1-x}\right]_{+}-\frac{8 \pi^{6}}{315} \delta(1-x), \\
& {\left[\mathrm{H}_{1,1,1,0}(x) \otimes\left[\frac{1}{1-x}\right]_{+}=-\mathrm{H}_{1,1,1,2}(x)-\mathrm{H}_{1,1,2,0}(x)-\mathrm{H}_{1,2,1,0}(x)-\mathrm{H}_{2,1,1,0}(x)\right.} \\
& \quad-\mathrm{H}_{1,1,1,0,0}(x)-3 \mathrm{H}_{1,1,1,1,0}(x)+\frac{\pi^{2}}{6} \mathrm{H}_{1,1,1}(x)-2 \zeta(3) \mathrm{H}_{1,1}(x)-\frac{\pi^{4}}{72} \mathrm{H}_{1}(x)
\end{aligned}
$$



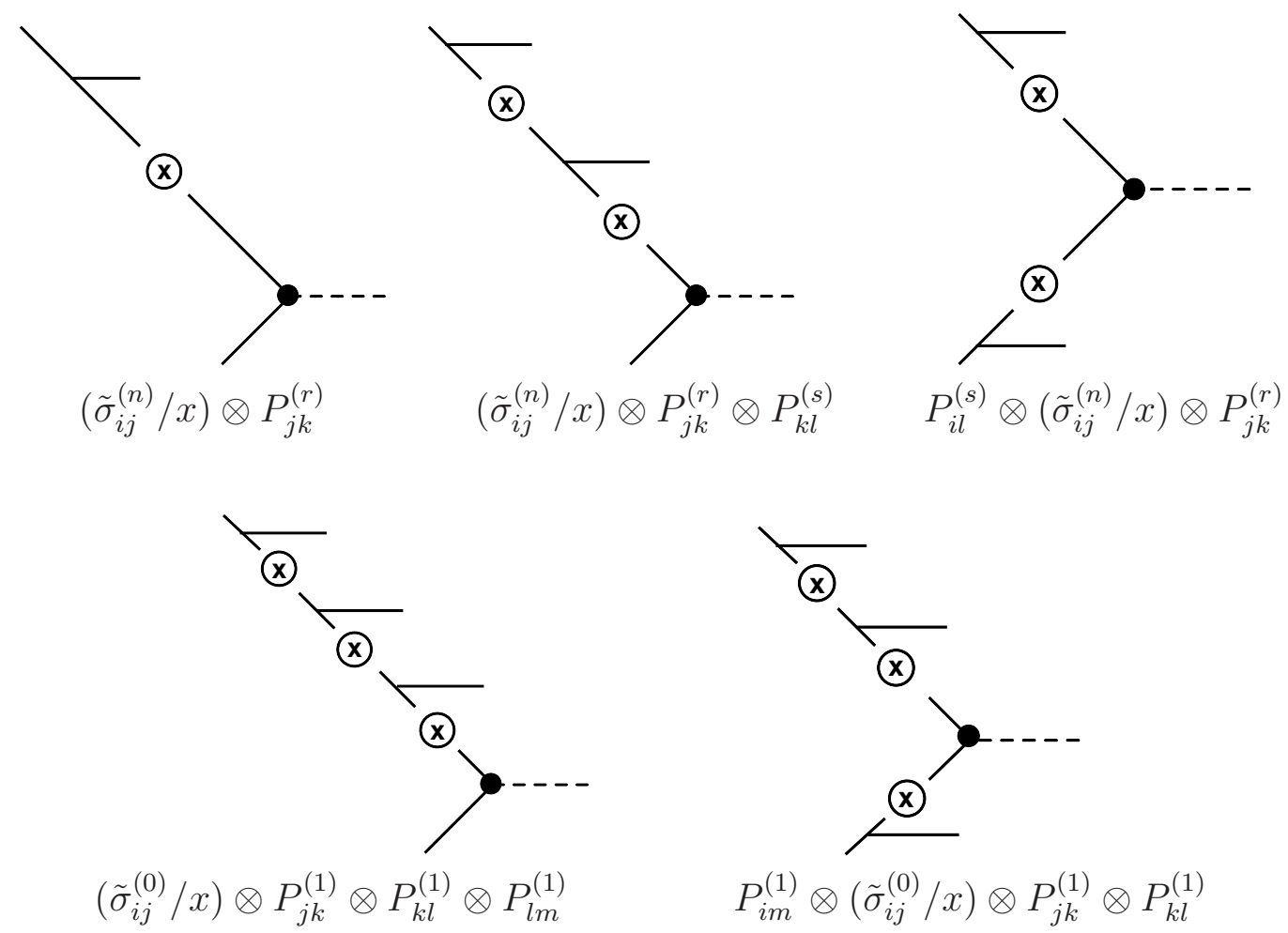

Figure 1: Schematic representation of the convolutions of the partonic cross section $\tilde{\sigma}_{i j}$ with the splitting functions $P_{j k}$.

$$
+\frac{\pi^{2}}{6} \zeta(3)-3 \zeta(5)
$$

\section{Summary}

In this paper we computed the NNLO partonic cross sections for the Higgs boson production to order $\epsilon$ which constitutes a building block for a $\mathrm{N}^{3} \mathrm{LO}$ calculation. Furthermore an algorithm has been developed which allows the evaluation of all convolutions of a partonic cross section with splitting functions needed at $\mathrm{N}^{3} \mathrm{LO}$. The described procedure might also be interesting for other processes like Drell-Yan production. We provide computer-readable results [12] both for the partonic cross sections and for all the convolutions entering the $\mathrm{N}^{3} \mathrm{LO}$ calculation of the partonic cross section for Higgs boson production at the LHC. 


\section{Acknowledgements}

This work was supported by the DFG through the SFB/TR 9 "Computational Particle Physics".

\section{A Convolutions and names of result files}

Tables 2 and 3 list the convolutions entering our calculation together with the names of the result files [12]. For convenience we use the notation $\bar{q} \equiv b$ and $q^{\prime} \equiv p$.

\section{References}

[1] G. Aad et al. [ATLAS Collaboration], Phys. Lett. B 716 (2012) 1 [arXiv:1207.7214 [hep-ex]].

[2] S. Chatrchyan et al. [CMS Collaboration], Phys. Lett. B 716 (2012) 30 [arXiv:1207.7235 [hep-ex]].

[3] S. Dittmaier, C. Mariotti, G. Passarino, R. Tanaka et al., [LHC Higgs Cross Section Working Group Collaboration], arXiv:1101.0593 [hep-ph].

[4] S. Dittmaier, C. Mariotti, G. Passarino, R. Tanaka et al., [LHC Higgs Cross Section Working Group Collaboration], arXiv:1201.3084 [hep-ph].

[5] A. Pak, M. Rogal and M. Steinhauser, JHEP 1109 (2011) 088 [arXiv:1107.3391 [hep-ph]].

[6] C. Anastasiou, S. Buehler, C. Duhr and F. Herzog, JHEP 1211 (2012) 062 [arXiv:1208.3130 [hep-ph]].

[7] R. V. Harlander and K. J. Ozeren, Phys. Lett. B 679 (2009) 467 [arXiv:0907.2997 [hep-ph]].

[8] A. Pak, M. Rogal and M. Steinhauser, Phys. Lett. B 679 (2009) 473 [arXiv:0907.2998 [hep-ph]].

[9] R. V. Harlander and K. J. Ozeren, JHEP 0911 (2009) 088 [arXiv:0909.3420 [hep-ph]].

[10] A. Pak, M. Rogal and M. Steinhauser, JHEP 1002 (2010) 025 [arXiv:0911.4662 [hep-ph]].

[11] R. V. Harlander, H. Mantler, S. Marzani and K. J. Ozeren, Eur. Phys. J. C 66 (2010) 359 [arXiv:0912.2104 [hep-ph]]. 


\begin{tabular}{|c|c|c|c|}
\hline 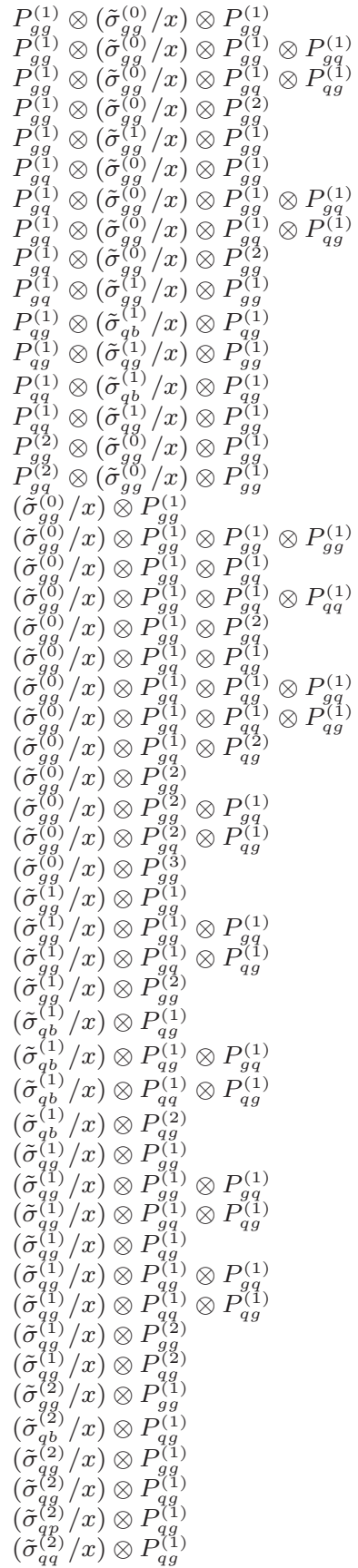 & $\begin{array}{l}\text { P1gg-Stil0gg-P1gg } \\
\text { P1gg-Stilogg-P1gg-P1gq } \\
\text { P1gg-Stil0gg-P1gq-P1qg } \\
\text { P1gg-Stil0gg-P2gg } \\
\text { P1gg-Stil1gg-P1gg } \\
\text { P1gq-Stil0gg-P1gg } \\
\text { P1gq-Stil0gg-P1gg-P1gq } \\
\text { P1gq-Stil0gg-P1gq-P1qg } \\
\text { P1gq-Stil0gg-P2gg } \\
\text { P1gq-Stil1gg-P1gg } \\
\text { P1qg-Stil1qb-P1qg } \\
\text { P1qg-Stil1qg-P1gg } \\
\text { P1qq-Stil1qb-P1qg } \\
\text { P1qq-Stil1qg-P1gg } \\
\text { P2gg-Stil0gg-P1gg } \\
\text { P2gq-Stil0gg-P1gg } \\
\text { Stil0gg-P1gg } \\
\text { Stil0gg-P1gg-P1gg-P1gg } \\
\text { Stil0gg-P1gg-P1gq } \\
\text { Stil0gg-P1gg-P1gq-P1qq } \\
\text { Stil0gg-P1gg-P2gq } \\
\text { Stil0gg-P1gq-P1qg } \\
\text { Stil0gg-P1gq-P1qg-P1gq } \\
\text { Stil0gg-P1gq-P1qq-P1qg } \\
\text { Stil0gg-P1gq-P2qg } \\
\text { Stil0gg-P2gg } \\
\text { Stil0gg-P2gg-P1gq } \\
\text { Stil0gg-P2gq-P1qg } \\
\text { Stil0gg-P3gg } \\
\text { Stil1gg-P1gg } \\
\text { Stil1gg-P1gg-P1gq } \\
\text { Stil1gg-P1gq-P1qg } \\
\text { Stil1gg-P2gg } \\
\text { Stil1qb-P1qg } \\
\text { Stil1qb-P1qg-P1gq } \\
\text { Stil1qb-P1qq-P1qg } \\
\text { Stil1qb-P2qg } \\
\text { Stil1qg-P1gg } \\
\text { Stil1qg-P1gg-P1gq } \\
\text { Stil1qg-P1gq-P1qg } \\
\text { Stil1qg-P1qg } \\
\text { Stil1qg-P1qg-P1gq } \\
\text { Stil1qg-P1qq-P1qg } \\
\text { Stil1qg-P2gg } \\
\text { Stil1qg-P2qg } \\
\text { Stil2gg-P1gg } \\
\text { Stil2qb-P1qg } \\
\text { Stil2qg-P1gg } \\
\text { Stil2qg-P1qg } \\
\text { Stil2qp-P1qg } \\
\text { Stil2qq-P1qg }\end{array}$ & 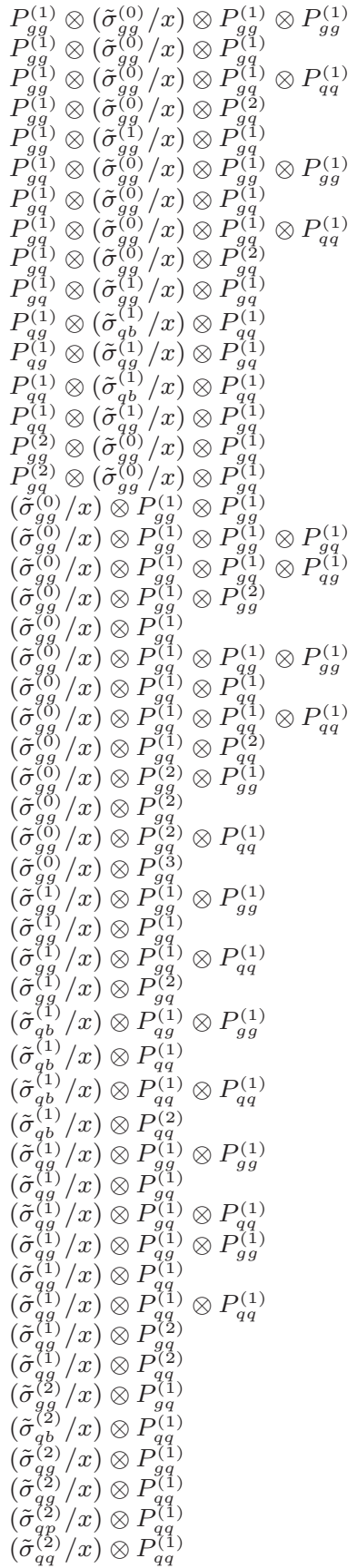 & $\begin{array}{l}\text { P1gg-Stil0gg-P1gg-P1gg } \\
\text { P1gg-Stilogg-P1gq } \\
\text { P1gg-Stil0gg-P1gq-P1qq } \\
\text { P1gg-Stil0gg-P2gq } \\
\text { P1gg-Stil1gg-P1gq } \\
\text { P1gq-Stil0gg-P1gg-P1gg } \\
\text { P1gq-Stil0gg-P1gq } \\
\text { P1gq-Stil0gg-P1gq-P1qq } \\
\text { P1gq-Stil0gg-P2gq } \\
\text { P1gq-Stil1gg-P1gq } \\
\text { P1qg-Stil1qb-P1qq } \\
\text { P1qg-Stil1qg-P1gq } \\
\text { P1qq-Stil1qb-P1qq } \\
\text { P1qq-Stil1qg-P1gq } \\
\text { P2gg-Stil0gg-P1gq } \\
\text { P2gq-Stil0gg-P1gq } \\
\text { Stil0gg-P1gg-P1gg } \\
\text { Stil0gg-P1gg-P1gg-P1gq } \\
\text { Stil0gg-P1gg-P1gq-P1qg } \\
\text { Stilogg-P1gg-P2gg } \\
\text { Stil0gg-P1gq } \\
\text { Stil0gg-P1gq-P1qg-P1gg } \\
\text { Stil0gg-P1gq-P1qq } \\
\text { Stil0gg-P1gq-P1qq-P1qq } \\
\text { Stil0gg-P1gq-P2qq } \\
\text { Stil0gg-P2gg-P1gg } \\
\text { Stil0gg-P2gq } \\
\text { Stil0gg-P2gq-P1qq } \\
\text { Stil0gg-P3gq } \\
\text { Stil1gg-P1gg-P1gg } \\
\text { Stil1gg-P1gq } \\
\text { Stil1gg-P1gq-P1qq } \\
\text { Stil1gg-P2gq } \\
\text { Stil1qb-P1qg-P1gg } \\
\text { Stil1qb-P1qq } \\
\text { Stil1qb-P1qq-P1qq } \\
\text { Stil1qb-P2qq } \\
\text { Stil1qg-P1gg-P1gg } \\
\text { Stil1qg-P1gq } \\
\text { Stil1qg-P1gq-P1qq } \\
\text { Stil1qg-P1qg-P1gg } \\
\text { Stil1qg-P1qq } \\
\text { Stil1qg-P1qq-P1qq } \\
\text { Stil1qg-P2gq } \\
\text { Stil1qg-P2qq } \\
\text { Stil2gg-P1gq } \\
\text { Stil2qb-P1qq } \\
\text { Stil2qg-P1gq } \\
\text { Stil2qg-P1qq } \\
\text { Stil2qp-P1qq } \\
\text { Stil2qq-P1qq }\end{array}$ \\
\hline
\end{tabular}

Table 2: Correlation of convolutions involving the partonic cross section and names of result file. 


\begin{tabular}{ll||ll}
\hline & & & \\
$P_{g g}^{(1)} \otimes P_{g g}^{(1)}$ & P1gg-P1gg & $P_{g g}^{(1)} \otimes P_{g g}^{(1)} \otimes P_{g g}^{(1)}$ & P1gg-P1gg-P1gg \\
$P_{g g}^{(1)} \otimes P_{g g}^{(1)} \otimes P_{g q}^{(1)}$ & P1gg-P1gg-P1gq & $P_{g g}^{(1)} \otimes P_{g q}^{(1)}$ & P1gg-P1gq \\
$P_{g g}^{(1)} \otimes P_{g q}^{(1)} \otimes P_{q g}^{(1)}$ & P1gg-P1gq-P1qg & $P_{g g}^{(1)} \otimes P_{g q}^{(1)} \otimes P_{q q}^{(1)}$ & P1gg-P1gq-P1qq \\
$P_{g g}^{(1)} \otimes P_{g g}^{(2)}$ & P1gg-P2gg & $P_{g g}^{(1)} \otimes P_{g q}^{(2)}$ & P1gg-P2gq \\
$P_{g q}^{(1)} \otimes P_{g g}^{(1)}$ & P1gq-P1gg & $P_{g q}^{(1)} \otimes P_{g g}^{(1)} \otimes P_{g g}^{(1)}$ & P1gq-P1gg-P1gg \\
$P_{g q}^{(1)} \otimes P_{g g}^{(1)} \otimes P_{g q}^{(1)}$ & P1gq-P1gg-P1gq & $P_{g q}^{(1)} \otimes P_{g q}^{(1)}$ & P1gq-P1gq \\
$P_{g q}^{(1)} \otimes P_{g q}^{(1)} \otimes P_{q g}^{(1)}$ & P1gq-P1gq-P1qg & $P_{g q}^{(1)} \otimes P_{g q}^{(1)} \otimes P_{q q}^{(1)}$ & P1gq-P1gq-P1qq \\
$P_{g q}^{(1)} \otimes P_{q g}^{(1)}$ & P1gq-P1qg & $P_{g q}^{(1)} \otimes P_{q g}^{(1)} \otimes P_{g g}^{(1)}$ & P1gq-P1qg-P1gg \\
$P_{g q}^{(1)} \otimes P_{q g}^{(1)} \otimes P_{g q}^{(1)}$ & P1gq-P1qg-P1gq & $P_{g q}^{(1)} \otimes P_{q q}^{(1)}$ & P1gq-P1qq \\
$P_{g q}^{(1)} \otimes P_{q q}^{(1)} \otimes P_{q g}^{(1)}$ & P1gq-P1qq-P1qg & $P_{g q}^{(1)} \otimes P_{q q}^{(1)} \otimes P_{q q}^{(1)}$ & P1gq-P1qq-P1qq \\
$P_{g q}^{(1)} \otimes P_{g g}^{(2)}$ & P1gq-P2gg & $P_{g q}^{(1)} \otimes P_{g q}^{(2)}$ & P1gq-P2gq \\
$P_{g q}^{(1)} \otimes P_{q g}^{(2)}$ & P1gq-P2qg & $P_{g q}^{(1)} \otimes P_{q q}^{(2)}$ & P1gq-P2qq \\
$P_{q g}^{(1)} \otimes P_{g g}^{(1)}$ & P1qg-P1gg & $P_{q g}^{(1)} \otimes P_{g q}^{(1)}$ & P1qg-P1gq \\
$P_{q g}^{(1)} \otimes P_{q g}^{(1)}$ & P1qg-P1qg & $P_{q q}^{(1)} \otimes P_{g g}^{(1)}$ & P1qq-P1gg \\
$P_{q q}^{(1)} \otimes P_{g q}^{(1)}$ & P1qq-P1gq & $P_{q q}^{(1)} \otimes P_{q g}^{(1)}$ & P1qq-P1qg \\
$P_{q q}^{(1)} \otimes P_{q q}^{(1)}$ & P1qq-P1qq & $P_{g g}^{(2)} \otimes P_{g g}^{(1)}$ & P2gg-P1gg \\
$P_{g g}^{(2)} \otimes P_{g q}^{(1)}$ & P2gg-P1gq & $P_{g q}^{(2)} \otimes P_{g g}^{(1)}$ & P2gq-P1gg \\
$P_{g q}^{(2)} \otimes P_{g q}^{(1)}$ & P2gq-P1gq & $P_{g q}^{(2)} \otimes P_{q g}^{(1)}$ & P2gq-P1qg \\
$P_{g q}^{(2)} \otimes P_{q q}^{(1)}$ & P2gq-P1qq & & \\
\hline & & & \\
\hline
\end{tabular}

Table 3: Correlation of convolutions involving only splitting functions and names of result file.

[12] http://www-ttp.particle.uni-karlsruhe.de/Progdata/ttp12/ttp12-45/

[13] G. Altarelli and G. Parisi, Nucl. Phys. B 126 (1977) 298.

[14] G. Curci, W. Furmanski and R. Petronzio, Nucl. Phys. B 175 (1980) 27.

[15] S. Moch, J. A. M. Vermaseren and A. Vogt, Nucl. Phys. B 688 (2004) 101 [hep$\mathrm{ph} / 0403192]$.

[16] A. Vogt, S. Moch and J. A. M. Vermaseren, Nucl. Phys. B 691 (2004) 129 [hep$\mathrm{ph} / 0404111]$.

[17] C. Anastasiou and K. Melnikov, Nucl. Phys. B 646 (2002) 220, arXiv:hepph/0207004.

[18] V. Ravindran, J. Smith and W. L. van Neerven, Nucl. Phys. B 665 (2003) 325, arXiv:hep-ph/0302135.

[19] S. Dawson, Nucl. Phys. B 359 (1991) 283.

[20] A. Djouadi, M. Spira and P. M. Zerwas, Phys. Lett. B 264 (1991) 440.

[21] M. Spira, A. Djouadi, D. Graudenz and P. M. Zerwas, Nucl. Phys. B 453 (1995) 17, arXiv:hep-ph/9504378. 
[22] R. V. Harlander and W. B. Kilgore, Phys. Rev. Lett. 88 (2002) 201801, arXiv:hepph/0201206.

[23] K. G. Chetyrkin, B. A. Kniehl and M. Steinhauser, Nucl. Phys. B 510 (1998) 61, arXiv:hep-ph/9708255.

[24] M. Kramer, E. Laenen and M. Spira, Nucl. Phys. B 511 (1998) 523 [arXiv:hep$\mathrm{ph} / 9611272]$.

[25] Y. Schröder and M. Steinhauser, JHEP 0601 (2006) 051, arXiv:hep-ph/0512058.

[26] K. G. Chetyrkin, J. H. Kühn and C. Sturm, Nucl. Phys. B 744 (2006) 121, arXiv:hep$\mathrm{ph} / 0512060$.

[27] J. Blumlein and S. Kurth, Phys. Rev. D 60 (1999) 014018 [arXiv:hep-ph/9810241].

[28] J. Ablinger, J. Blumlein and C. Schneider, J. Math. Phys. 52 (2011) 102301 [arXiv:1105.6063 [math-ph]].

[29] J. A. M. Vermaseren, Int. J. Mod. Phys. A 14 (1999) 2037 [hep-ph/9806280].

[30] E. Remiddi and J. A. M. Vermaseren, Int. J. Mod. Phys. A 15 (2000) 725 [arXiv:hepph/9905237].

[31] http://www.nikhef.nl/ form/maindir/packages/harmpol/

[32] D. Maitre, Comput. Phys. Commun. 174 (2006) 222 [arXiv:hep-ph/0507152].

[33] D. Maitre, Comput. Phys. Commun. 183 (2012) 846 [hep-ph/0703052 [HEP-PH]].

[34] C. Anastasiou, K. Melnikov and F. Petriello, Nucl. Phys. B 724 (2005) 197 [hepph/0501130].

[35] A. G. -D. Ridder, T. Gehrmann, E. W. N. Glover and J. Pires, arXiv:1211.2710 [hep-ph]. 